\title{
Study of Stable Cathodes and Electrolytes for High Specific Density Lithium-Air Battery
}

Dionne M. Hernández-Lugo ${ }^{1}$, James Wu', William Bennett', Yu Ming' ${ }^{2}$, Yu Zhu²

Photovoltaics and Electrochemical Systems Branch, NASA Glenn Research Center, 309-1, Cleveland, Ohio 44135 NASA Department of Polymer Science, The University of Akron, Akron, Ohio 44325-3909

dionne.m.hernandez-lugo-1@nasa.gov, Tel. 216-433-5911

\section{Abstract}

Future NASA missions require high specific energy battery technologies, > $400 \mathrm{Wh} / \mathrm{kg}$. Current NASA missions are using "state-of-the-art" (SOA) Li-ion batteries (LIB), which consist of a metal oxide cathode, a graphite anode and an organic electrolyte. NASA Glenn Research Center is currently studying the physical and electrochemical properties of the anode-electrolyte interface for ionic liquid based Li-air batteries. The voltage-time profiles for Pyr13FSI and Pyr14TFSI ionic liquids electrolytes studies on symmetric cells show low over-potentials and no dendritic lithium morphology. Cyclic voltammetry measurements indicate that these ionic liquids have a wide electrochemical window. As a continuation of this work, $\mathbf{s p}^{2}$ carbon cathode and these low flammability electrolytes were paired and the physical and electrochemical properties were studied in a Li-air battery system under an oxygen environment.

\section{Applications}
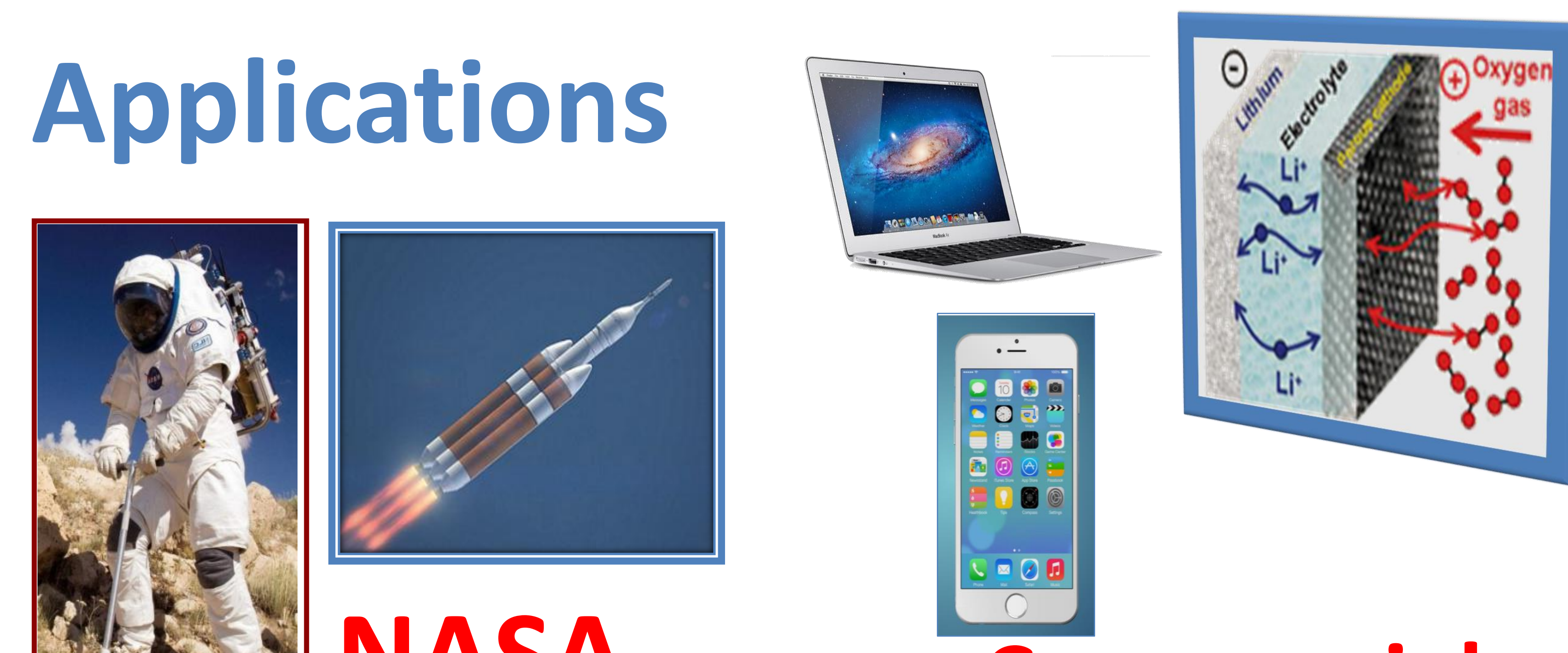

Commercial
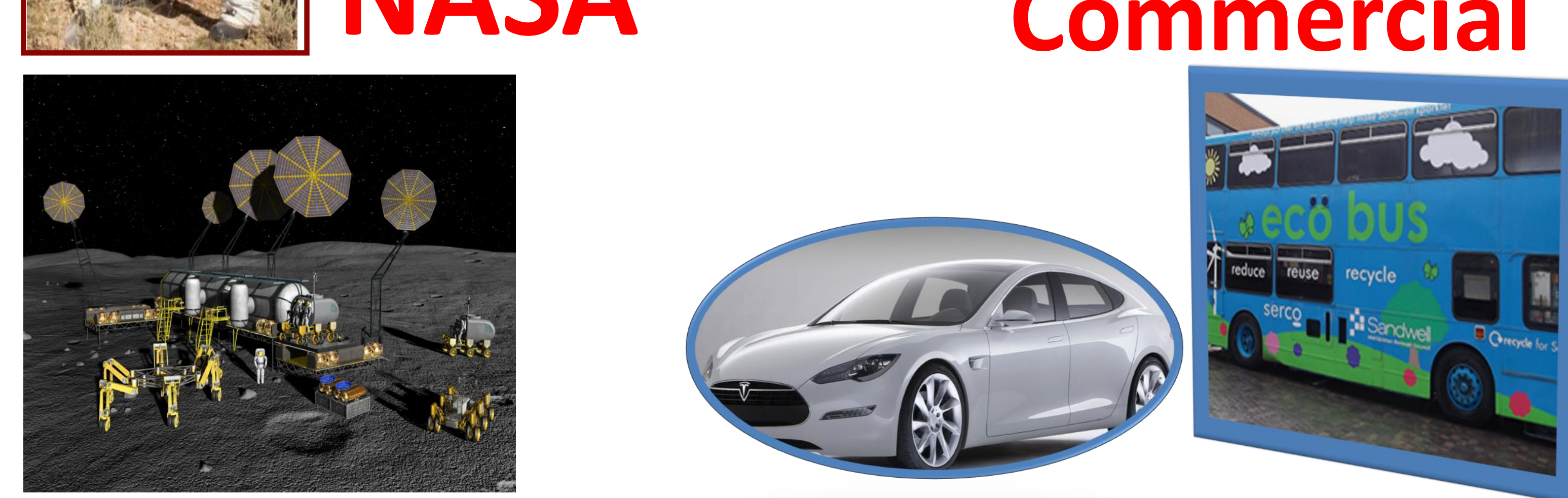

Why Lithium Metal?

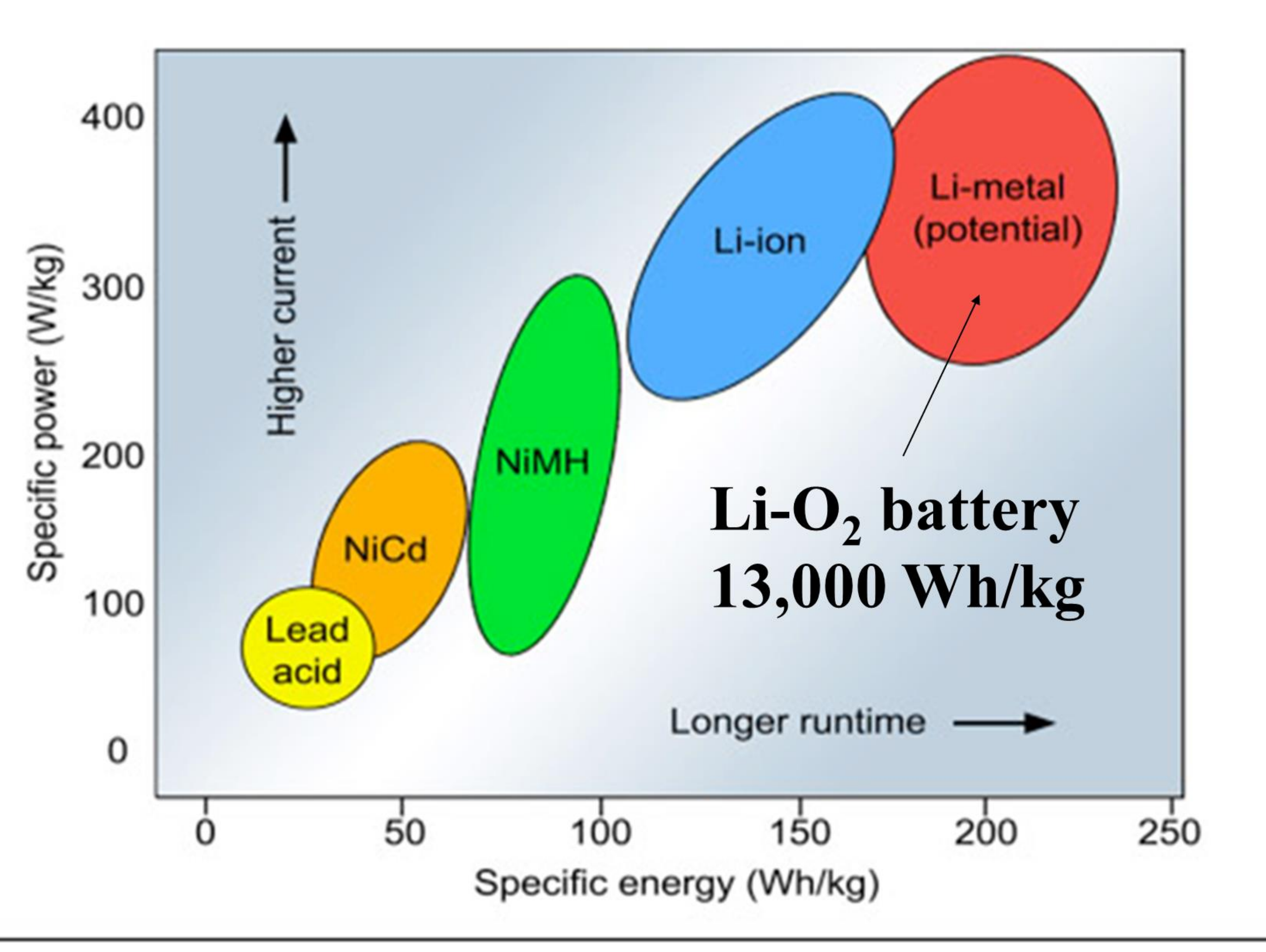

Vertically Aligned -CNT cathode

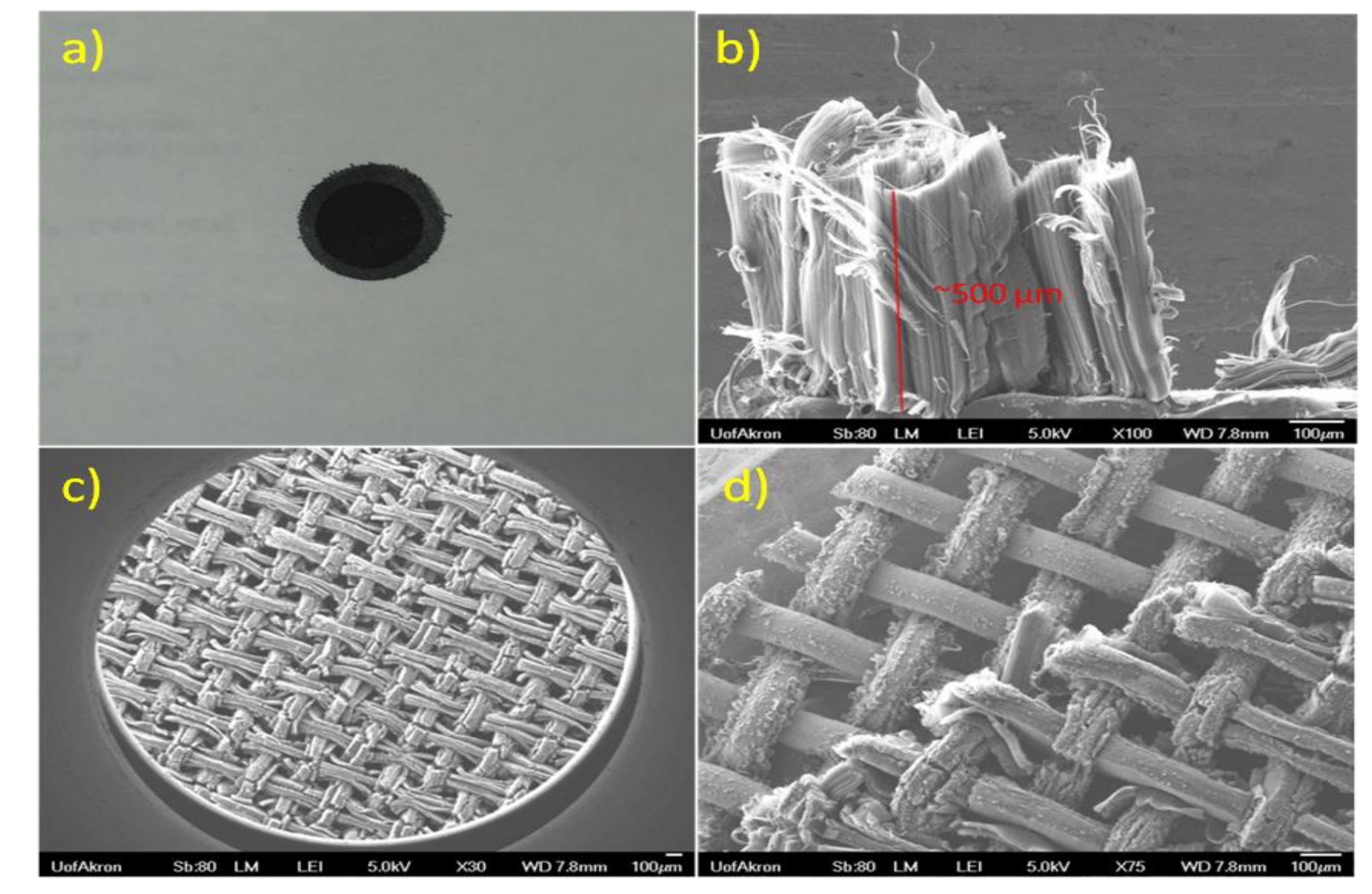

Vertically aligned carbon nanotubes (VACNT) directly grown on stainless steel mesh. The VACNT have a height of $500 \mu \mathrm{m}$. U. of Akron

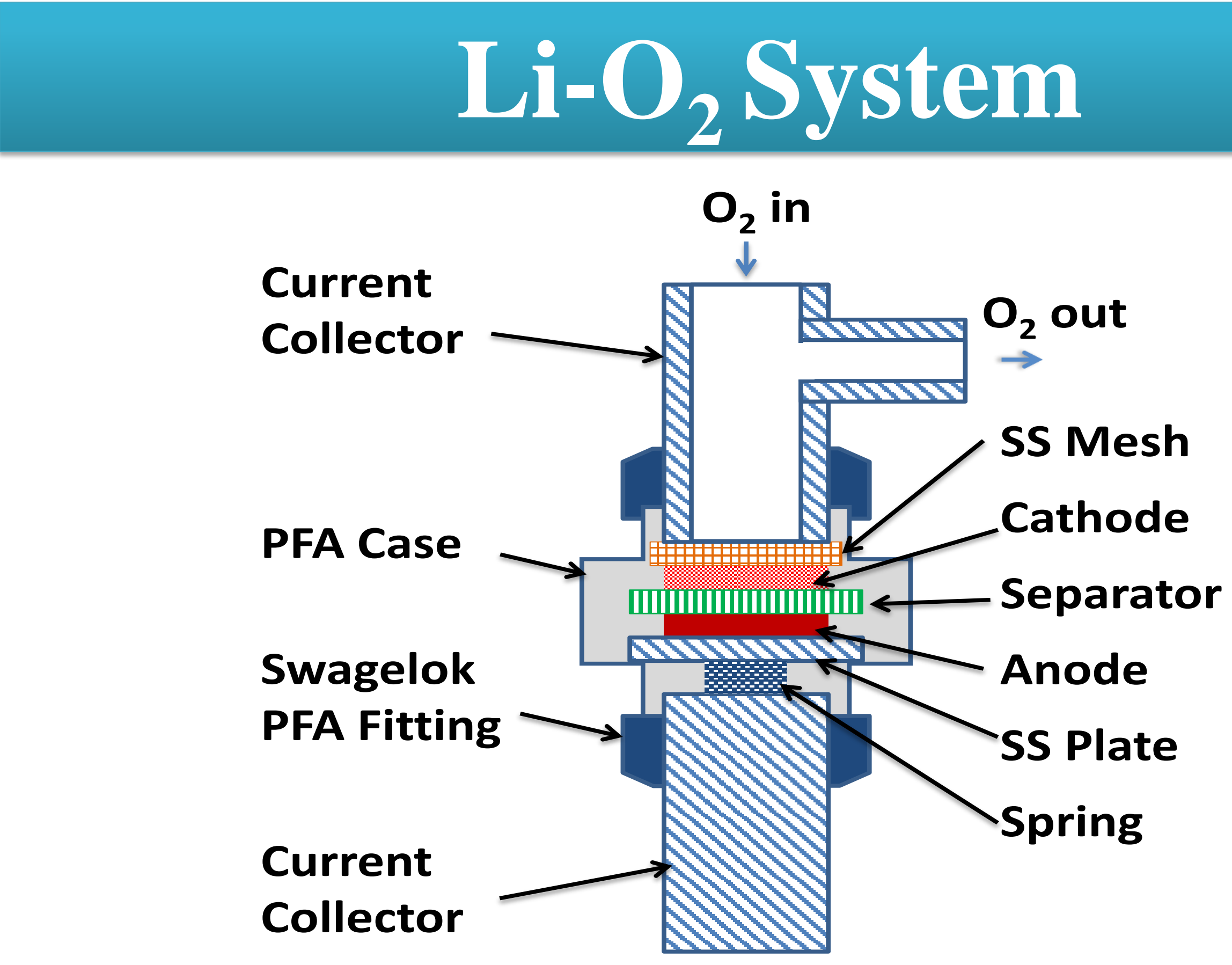

Ionic Liquids Characteristics

Low flammability

$>$ Thermally and electrochemically stable

Suppress dendrite formation vs. traditional organic electrolytes

Physical Properties of Electrolytes

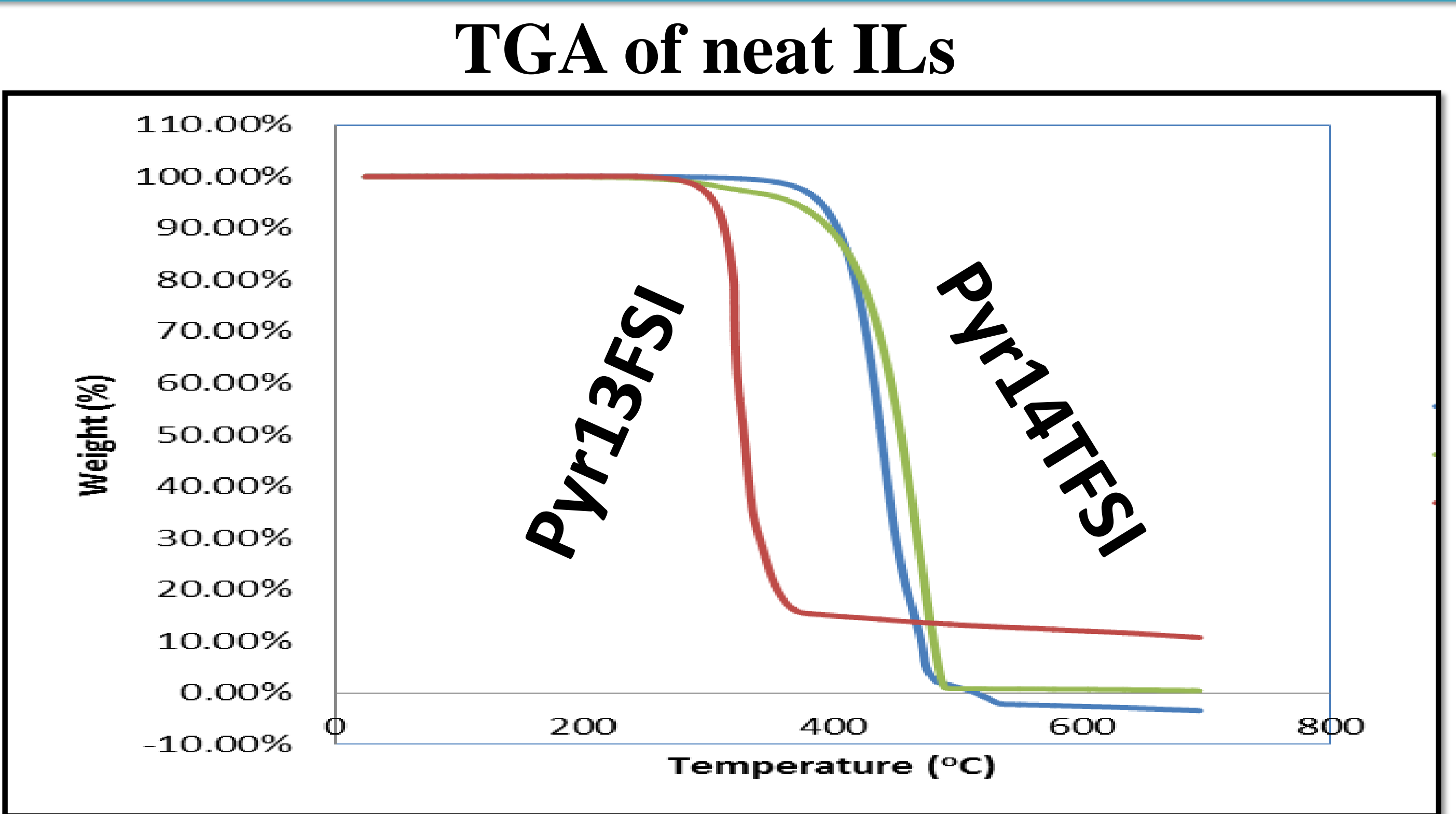

ILs show a decomposition temperature $>300^{\circ} \mathrm{C}$.

\section{Electrochemical Characterization}
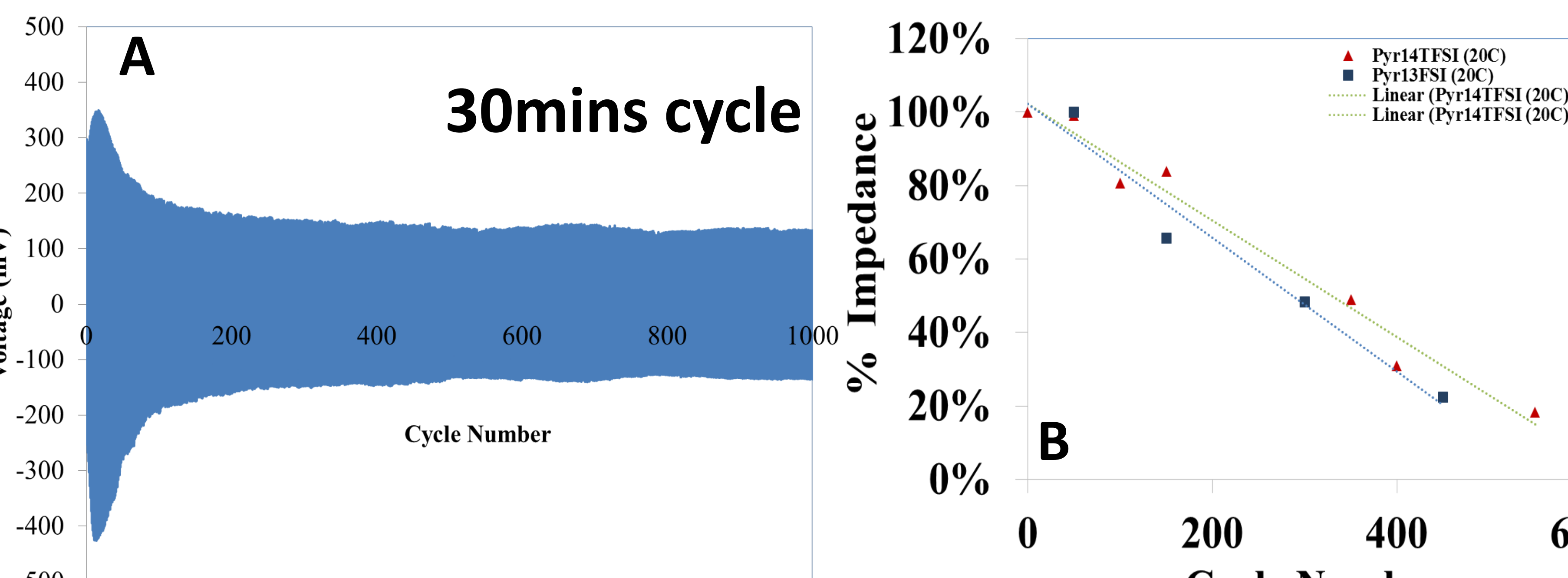

A. Voltage vs. Cycle Number of $0.5 \mathrm{~m}$ LiTFSI in Pyr14TFSI symmetric cycling at $1 \mathrm{~mA} / \mathrm{cm} 2,30$ mins cycle. B. \% Impedance vs. cycle number for $0.5 \mathrm{~m}$ LiTFSI in Pyr14TFSI and $0.5 \mathrm{~m}$ LiFSI in Pyr13FSI
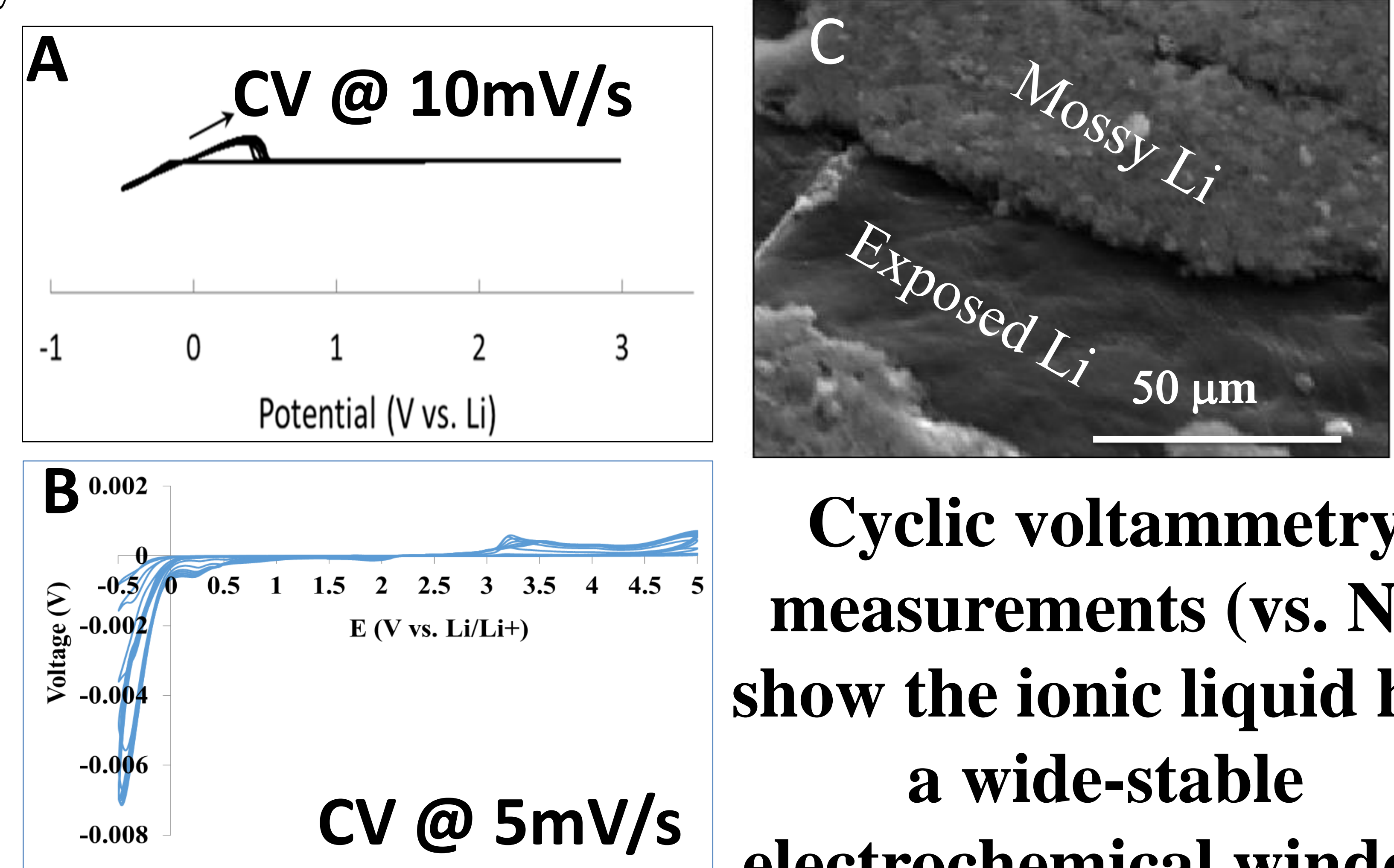

Cyclic voltammetry measurements (vs. Ni) show the ionic liquid has a wide-stable electrochemical window

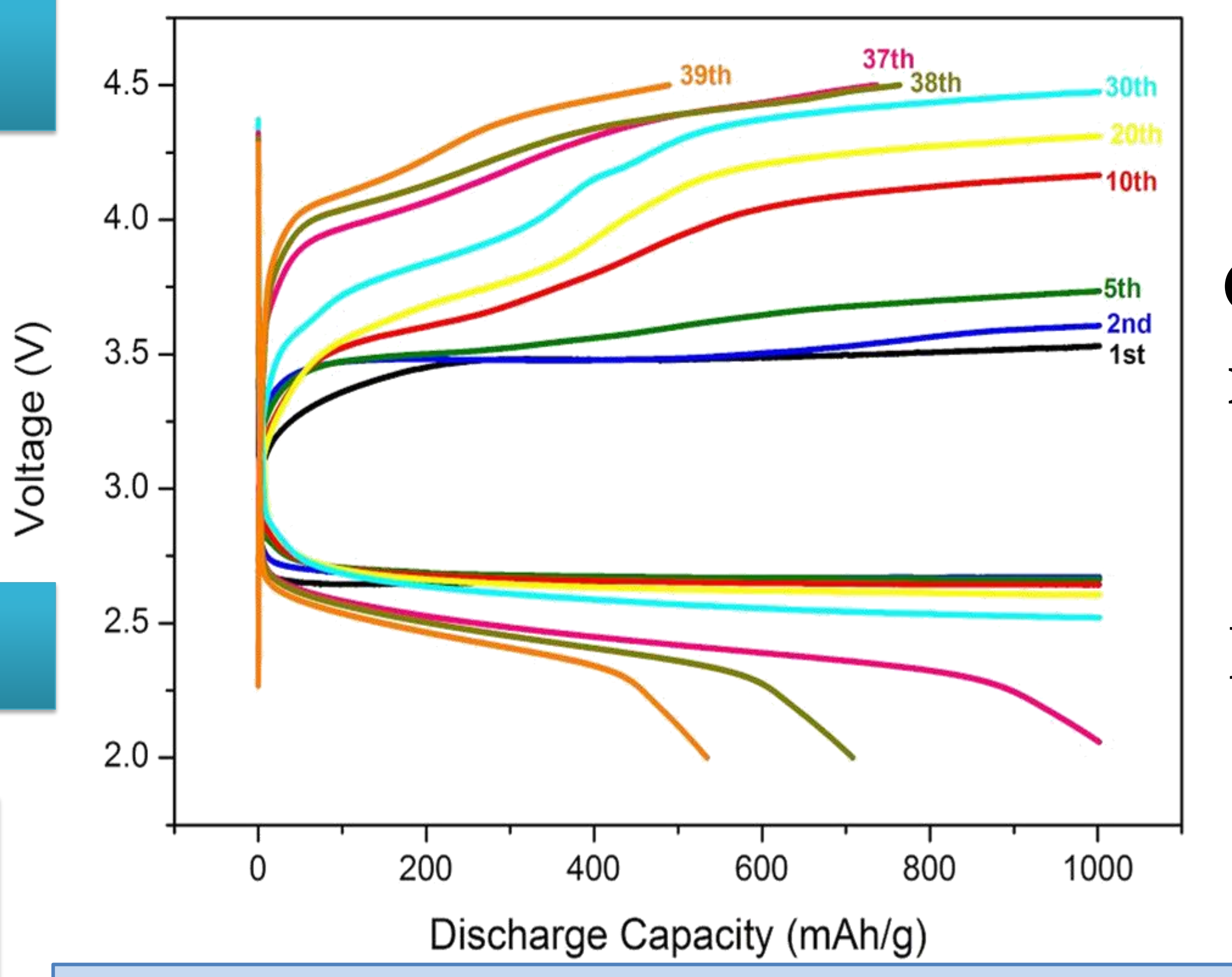

Vertically AlignedCNT cathode tested in an in-house built Li-O2 system with The (1:1 v/v) NMP LiTFSI/Pyr14TFSI electrolyte U. of Akron

\section{Summary}

1. The air cathode demonstrated rechargeable cycles for 40 cycles with $1000 \mathrm{mAh} / \mathrm{g}$.

2. [Pyr14][TFSI] and [Pyr13][FSI] have decreasing resistance (increasing Li surface area).

3. SEM images at 1000 cycles show no dendrites.

4. Air cathodes with other compositions will be synthetize to enhance the cycle life. 